\title{
Efficient Edit Propagation Using Hierarchical Data Structure
}

\author{
Chunxia Xiao, Yongwei Nie, Feng Tang
}

\begin{abstract}
This paper presents a novel unified hierarchical structure for scalable edit propagation. Our method is based on the key observation that in edit propagation, appearance varies very smoothly in those regions where the appearance is different from the userspecified pixels. Uniformly sampling in these regions leads to redundant computation. We propose to use a quadtree based adaptive subdivision method such that more samples are selected in similar regions and less in those that are different from the user-specified regions. As the result, both the computation and memory requirement is significantly reduced. In edit propagation, an edge-preserving propagation function is firstly built, and the full solution for all the pixels can be computed by interpolating from the solution obtained from the adaptively-subdivided domain. Furthermore, our approach can be easily extended to accelerate video edit propagation using an adaptive octree structure. In order to improve user interaction, we introduce several new Gaussian Mixture Model (GMM) brushes to find pixels that are similar to the user specified regions. Compared with previous methods, our approach requires significantly less time and memory, while achieving visually same results. Experimental results demonstrate the efficiency and effectiveness of our approach on high resolution photographs and videos.
\end{abstract}

Index Terms-Tone adjustment, Gaussian mixture model, hierarchical Data Structure, high dynamic range imaging, tone mapping

\section{INTRODUCTION}

Once photographs are generated from digital cameras, they usually need to go through a series of local adjustments, for example, correction and enhancement operations, to be visually appealing. Many image editing softwares such as Adobe Photoshop [1] have presented a variety of powerful adjustment tools for manipulating photographs. However, these manipulation tools are usually tedious and time-consuming even for professionals. Recently, several stroke-based edit propagation approaches have been presented [2], [3], [4]. In these systems adjustments in selected regions are propagated to the entire image using edge-preserving optimization methods. These methods typically involve solving large linear systems which have significant computation and space requirements. This limits their applications on high resolution images and videos. Although several researchers [2], [4] have tried to address this problem, improvements are still needed for time and space-critical applications, such as edit propagation for high resolution images and video sequences.

Inspired by methods in image composition [5] and large-scale fluid simulation [6] which use adaptive resolution to efficiently solve large linear systems, we present a hierarchical approach for scalable edit propagation. Our approach is based on the key observation that the appearance of many large regions in the image are

- Chunxia Xiao and Yongwei Nie are with the School of Computer, Wuhan University, Wuhan China. Email: cxxiao@whu.edu.cn, nieyongwei@gmail.com.

Feng Tang is a researcher in the Multimedia Interaction and Understanding Lab (MIUL) of HP Labs in Palo Alto, CA. Email: feng.tang@hp.com Corresponding author: Chunxia Xiao,Email: cxxiao@whu.edu.cn. different from the specified region. However it can be observed, in constraints based edit propagation [2] the user's appearance constraints have less influence in these areas. The difference between the original image and its associated accurate edit propagation results resolved using full solution is usually small. Thus, representing each pixel in these regions with one variable may not be necessary. These areas can be accurately interpolated with fewer variables from a larger region of support using an hierarchical representation.

With this idea in mind, we propose a hierarchical approach which subdivides the image using quadtree such that more samples are selected in regions that are similar to the user specified regions while less in those that are different (Fig.1(c)). In some situations when the regions similar to the user specified parts occupy a large part of the image (Fig.1(b)), the solution is is usually smooth. We use hierarchical quadtree in these similar regions based on the edges information. High resolution samples are applied near the strong edges and lower resolutions in areas farther away from these edges (Fig.1(e)). This is especially useful for edge-preserving edit propagation. With this technique, the solution space is significantly reduced, and edges are preserved during the propagation. Then we build an edge-preserving edit propagation function in this reduced space, and the full solution of the entire image is interpolated from the solution of the reduced space.

In our approach, the hierarchical data structure is defined based on the similarity measure between each pixels and user specified regions, so an effective similarity measure is critical to our approach. We define several probabilistic representations - GMM (Gaussian Mixture Model) brushes for this purpose. This representation 
is especially effective in selecting similar appearance regions, without the need for the user to specify all the pixels to be editted. In addition it can preserve underlying appearance features such as image edges(Fig.1(b)).

With this hierarchical data structure, full resolution edit propagation can be approximated by solving a linear system of size $O(p)$, where $p$ is the total length of the edges of the similar regions. For typical cases, $p$ is $O(\sqrt{n})$ where $n$ is the number of pixels in the image. The time-consuming full solution space is reduced to a significantly reduced space, which requires much less computation and memory. Since the editing is propagated according to the adaptive quadtrees guided by the similarity measure, more samples are selected in the regions sensitive to edit propagation such as boundaries. This makes the full solution interpolated from the reduced solution visual identical to that of using the original solution.

We further demonstrate the efficiency of our method by performing edit propagation in high-resolution videos. We propose an efficient energy optimization function that incorporates space-time information to propagate user edits. An adaptive octree structure is used to speed up the process. To the best of our knowledge, we are the first to present an effective approach to perform video edit propagation that constrained edits are propagated to the spatially and temporally close regions with similar appearance. It should be noted that the developed hierarchy based constraint propagation approach is a general one, and can be used to interactively apply a variety of other adjustments such as exposure, contrast, saturation, measured materials.

This paper makes the following contributions:

1) a multi-GMM brush is introduced to effectively compute the similarity field in finding regions that are similar to user specified pixels and construct adaptive hierarchical data structure;

2) an efficient edit propagation algorithm based on the adaptive quadtree structure, which makes both the computation and memory requirement significantly reduced, while still achieving visually appealing results;

3) an efficient video edit propagation algorithm using the octree data structure, which is very efficient and scalable to process gigapixel videos.

The rest of our paper is organized as follows. Section II reviews related work. Section III describes fast image edit propagation using hierarchical data structure, and also gives several efficient brushes for similar regions selection. In section IV, we present fast edit propagation algorithms for video. In section $\mathrm{V}$, computational complexity analysis is presented, and in section VI, we show the experimental results and limitation of our methods. The paper is concluded in section VII.

\section{Related work}

In traditional image editing systems, the user needs to precisely identify which region of an image needs to be edited, this process usually requires tedious user interaction. Recently stroke based interfaces have been proposed which only require the user to roughly specify the regions of interest using several strokes. These regions are used as examples for image editing and the system can automatically find regions that the user wants the edit to affect. By using the proposed edit propagation methods [2], [3], [4], [7], [8], the constraints set by the user's strokes are propagated to the entire image in a piecewise smooth manner. These methods have been proved effective for images [2], [4], [7], [8] as well as materials edit propagation on 3D surfaces [3].

To perform edit propagation and local appearance manipulation [2], [3], [4], the first step is to identify the regions that the user intend to adjust. As shown in Fig.2, the user is adjusting the color of the tulips in the image. Since there are so many isolated tulips, it would be very time-consuming to stroke all of them. To address this problem, Lischinski et al. [2] presented a luminance brush that selects the image pixels whose luminance fall within a global threshold to those pixels under user painted strokes. Since luminance brush is a simple monochrome image brush, it is not effective in many cases, for example, it will select many scattered pixels and meaningless regions, as illustrated in Fig.2. As an alternative method, Li et al. [8] built a boostingbased classification for similar region selection. Although better results than luminance brush [2] were achieved, in some cases the selected results may not be consistent and many scatted pixels may appeared, as illustrated in Fig.9 and Fig.10. To obtain better results, much postprocessing work has to be done [8].

Stroke-based interface has been widely used in foreground extraction [9], [10], [11], [12], [13] and video completion [14]. Stroke based similar region selection used in edit propagation is closely related to interactive image segmentation [9], [15], [16]. However, the goal of image segmentation is to obtain coherent regions in binary style, while the similarity computation is to compute the appearance similarity between the pixel and stroked pixels, the results are fractal. In this way, it is more related to the alpha image matting [10], [15], but appearance similarity prefer to select all pixels in the image that are close in appearance, even they are not spatially close. So many isolated regions may be selected, as illustrated in Fig.10. The computed similarity results are used to smoothly interpolate adjusted color or tone on the stroked pixels, and to achieve the longrange and soft transition of appearance edit in the entire image. To make effective selection for appearance similar regions, different from the existing methods [2], [8], we present new GMM brushes which are applicable to both monotonic and color images.

Lischinski et al. [2] presented an optimization method to propagate local tonal adjustment to all similar regions in the images, and attractive results are obtained. However, this algorithm needs to solve a large linear system, which suffers from the time complexity and 


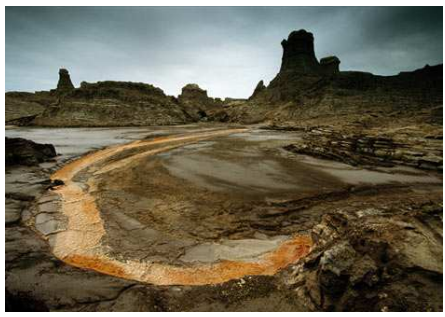

(a)

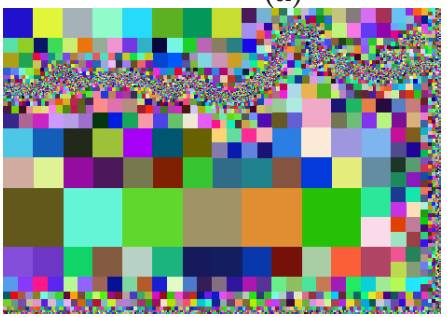

(e)

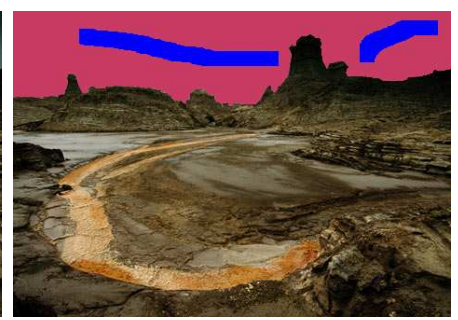

(b)

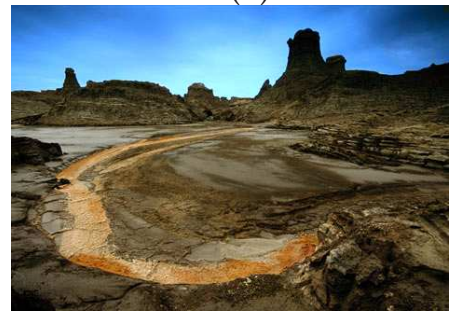

(f)

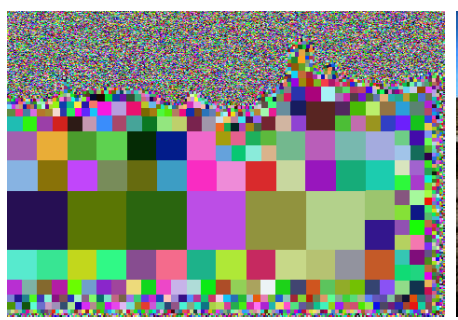

(c)

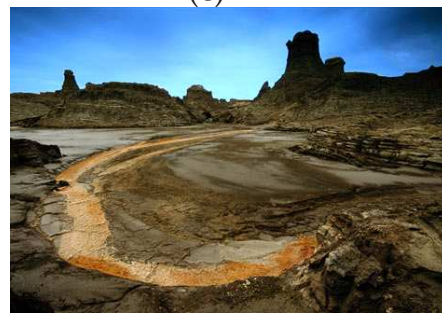

(g)

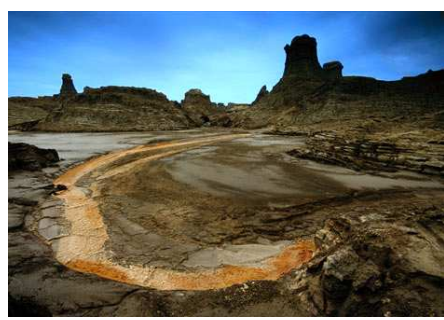

(d)

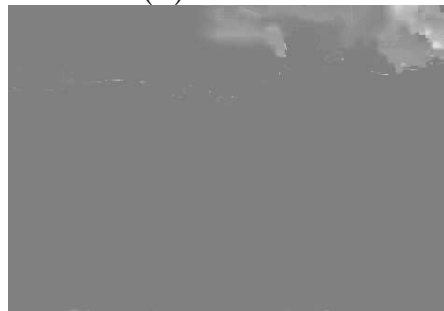

(h)

Fig. 1. Our edit propagation framework. (a) Original image, (b) strokes are drawn on (a) and similar regions are selected (red regions) using GMM similarity measure, (c) partial quadtree decomposition (PQD) of (a) based on the similarity field (b), high resolution is used at the similar regions, (d) edit propagation results computed on quadtree (c), (e) complete quadtree decomposition (CQD) of (a) based on the similarity measure (b), where the similar regions are also further subdivided according to the edges information, (f) edit propagation results computed on quadtree (e), (g) the full resolution results of (b), (h) the visualization of error between the solution (f) computed using the reduced linear system and the solution (g) using full linear system.

memory requirement. This makes it not practical to process multi-megapixel images. Many efficient algorithms have been proposed to accelerate the solution of large linear systems. For example, large linear systems can be solved efficiently using multigrid method [17] and this method also can be adapted to the GPU [18]. Szeliski at al. [19] introduced a preconditioner that significantly accelerates the convergence of an iterative conjugate gradient solver. However, all these methods do not address the fundamental scalability problem: they still require solving linear systems in the order of the number of pixels, and the $O(n)$ memory required by a sparse solver is impractical when processing multi-megapixel digital imagery.

To solve the scalability problem in edit propagation, as well as reducing the memory requirement, we propose a hierarchical data structure to significantly reduce the computational complexity. There are several existing methods on using adaptive resolution in solving large linear systems or discretized partial differential equations. Szeliski and Shum [20] used quadtrees for hierarchical motion estimation in video; Losasso et al. [6] performed large-scale fluid simulations by solving the Poisson equation on adaptive octree grids. Agarwala [5] showed how quadtrees can be applied to efficient gradient-domain composition by transforming the problem into a reduced space to make composition effective. Our formulation is inspired by these approaches, and is more general in its support for user edits.

Several other image edit propagation methods also have been proposed. An at al. [4] presented an edit propagation algorithm which enforces the constraints defined over all pairs of points in the dataset. To efficiently solve the derived dense matrix, they proposed an approximate algorithm which uses the inherent structure of the matrix. The limitation of the approach is that the scalability of this method is limited by the amount of memory required to store the sampling matrix. Instead of solving a piecewise smooth interpolation in pixel accuracy [2], Chen at al. [21] presented an interactive edge-aware method which used a scalar bilateral grid to achieve similar results. Although the method can run in real-time during the selection, compared to image-pixel based approaches [2], [7], this method does not work well at pixel level.

As one concurrent work [22], Xu et al. proposed a similar accelerated edit propagation method using adaptive kd-tree, while our method uses an adaptive quadtree for image and octree for video. There are two main differences between these two methods. The first is the similarity measure definition used to find regions that need to be propagated. Our approach uses proposed multi-GMM probability value, while $\mathrm{Xu}$ et al. [22] uses the similarity measure of [4] as a function of the distance between the points of spatial locations and their appearance vectors [4]. Our method is more accurate in computing the similarity map. The second difference is that $\mathrm{Xu}$ et al. [22] do not use dense samples in the more similar regions, which is similar to our partly quadtree decomposition (PQD) method. Using our further proposed complete quadtree decomposition (CQD), we build a adaptive quadtree in these similar regions, which is 
faster in situations when the regions that are similar to the user selection occupy a large part of the image. As shown in the section VI, our method presents faster and better results. Fattal [23] proposed edge-avoiding wavelets for image editing, they presented a family of second-generation wavelets constructed using dataprediction lifting schemes, and these wavelets adapt to the edge content of the image. Multi-scale representation based on these wavelets can speedup edge-preserving image operations including edit propagation. However, this method can not receive satisfied edit propagation quality.

\section{IMAGE EDIT PROPAGATION}

Our goal is to present a computationally efficient solution to adjust properties of an image in a spatially varying manner. In our framework, the user is not required to specify all the regions they want to adjust. Instead, the user only needs to specify some example regions and apply edits. These edits are then propagated to the entire image via a constrained edge-preserving energy minimization function. Our overall algorithm consists of the following four stages.

1) In the input stage, the user scribbles to roughly specify the example regions that need to be adjusted. These regions serve as the hard constraints and are used to compute the the similarity between each pixel and user selected regions.

2) In the second stage, based on the appearance similarity and other properties such as image edges, a hierarchical data structure (quadtree) is constructed. It is used to reduce the computational complexity for the processing of the entire image.

3) The third stage is the solution of the linear system derived from the energy minimization function for edge preserving edit propagation. This function is defined on the efficient quadtree data structure, and the solution in the reduced space is obtained.

4) In the final output stage, the reduced space solution is interpolated to obtain the full solution for the entire image.

The proposed approach is highly efficient, users are provided with (near) realtime previews of overall results, even with high-resolution video. In the following several subsections we will present technical details for each of the above mentioned stages.

\subsection{Region selection}

We present a new GMM (Gaussian mixture model) brush which is capable of handling both monotonic and color image similarity measure between the user selected pixels and the rest pixels to identify those regions that are similar to user scribbled ones.
GMM is a widely used model in image analysis [15] for the description of color distribution. GMM is a probability density composed of a set of Gaussian models. We construct a GMM on the pixel colors of user specified regions, and GMM is modeled using a full-covariance Gaussian mixture with $\mathrm{K}$ components (typically $\mathrm{K}=5$ ). The GMM with K Gaussian models is defined as: $G(\vec{x})=$ $\sum_{k=1}^{K} p_{k} g_{k}(\vec{x})$, where $\vec{x}$ is D dimension vector, $p_{k}$ is the mixing coefficients satisfying $\sum_{k=1}^{K} p_{k}=1$, and $g_{k}(\vec{x})$ is Gaussian density function. To make the computation more robust, the GMM is performed in the log domain. Using the GMM model as the image representation, we present two brushes for region selection.

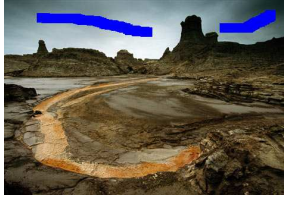

(a)

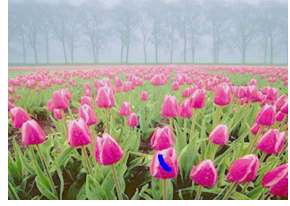

(d)

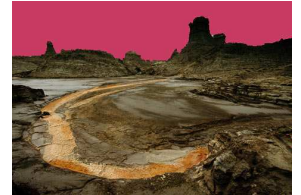

(b)

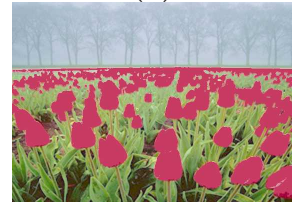

(e)

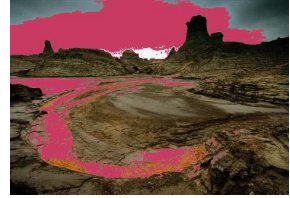

(c)

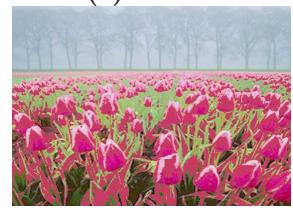

(f)
Fig. 2. Similar region selection using different brushes. (a)(d) Strokes drawn on the image, (b)(e) selected similar regions using GMM brush, (c)(f) selected similar regions using Luminance brush [2].

GMM brush: We use strokes to specify both foreground $F$ and background regions $B$ from the image. Let $F=\left\{\overrightarrow{x_{1}}, \overrightarrow{x_{2}}, \cdots, \overrightarrow{x_{N}}\right\}$ be the user selected pixels to be edited, which we call foreground. For each $x_{i} \in F$, we compute its probability value $G\left(x_{i}\right)$. We denote $\mu$ as the mean probability value of all pixels in $F$. The similarity between pixel $p$ outside of $F$ and the user specified region (represented using GMM) is defined as $\omega(p)=G(p)$.

We then build GMM models for both foreground and background regions. Let $\omega_{F}(p)$ and $\omega_{B}(p)$ be the probability value of pixel $p$ in $F$ and $B$, respectively, and $\mu_{F}$ and $\mu_{B}$ be the mean probability value of all pixels in $F$ and $B$.

The confidence that pixel $p$ being foreground can be evaluated as

$$
H(p)=\alpha \cdot\left|\omega_{F}(p)-\mu_{F}\right|+\beta \cdot\left|\omega_{B}(p)-\mu_{B}\right|,
$$

where the weight $\alpha$ is defined as

$$
\alpha=\frac{\sum_{i \in F \cup B} \phi_{j}^{F} \nu_{j}^{F} z_{j}}{\sum_{i \in F \cup B} \phi_{j}^{F} z_{j}},
$$

and $\phi_{j}^{F}=\exp \left(-\left|\omega_{F}(p)-\omega_{F}\left(p_{j}\right)\right|^{2}\right), \nu_{j}^{F}=\exp \left(-\mid \omega_{F}\left(p_{j}\right)-\right.$ $\left.\left.\mu_{F}\right|^{2}\right)$; the weight $\beta$ is defined in a similar way. The pixel label $z$ is defined as $z_{j}=1$ if $j \in F$, and $z_{j}=-1$ if $j \in B$. The weights $\alpha$ and $\beta$ are defined as the weighted sum 


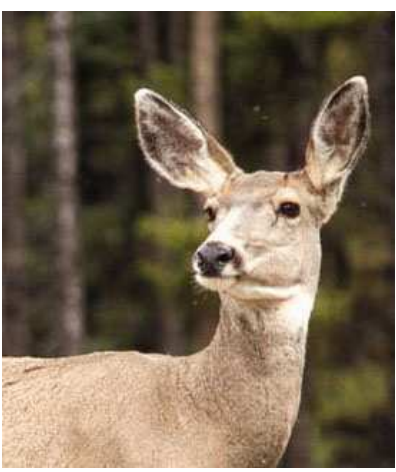

(a)

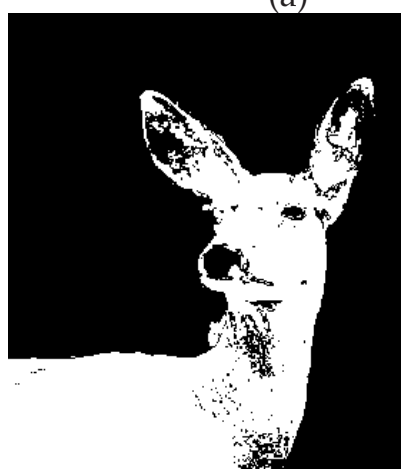

(e)

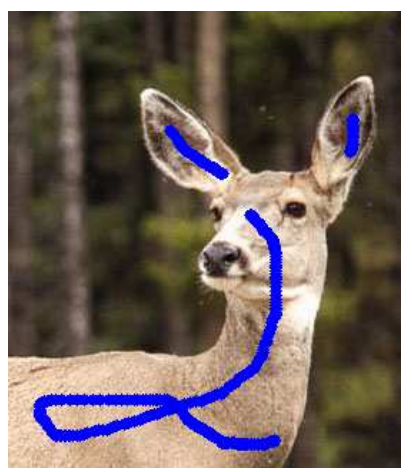

(b)

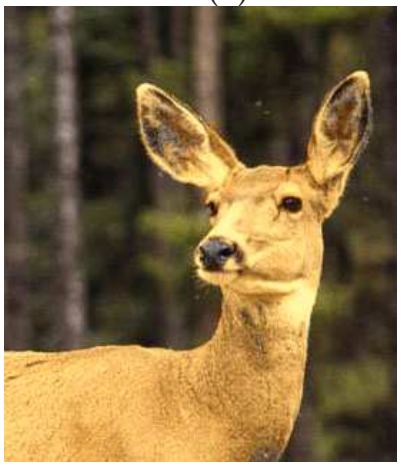

(f)

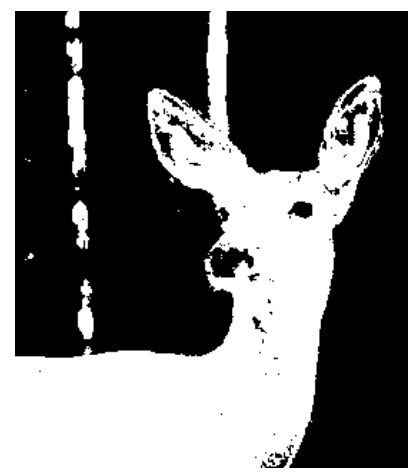

(c)

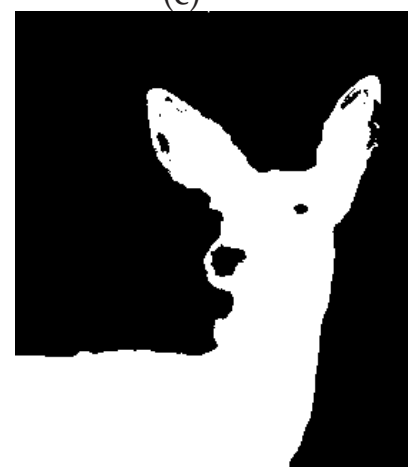

(g)

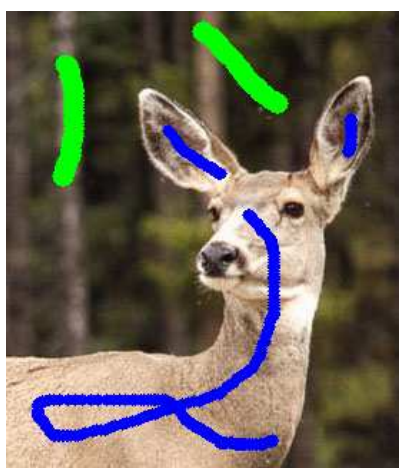

(d)

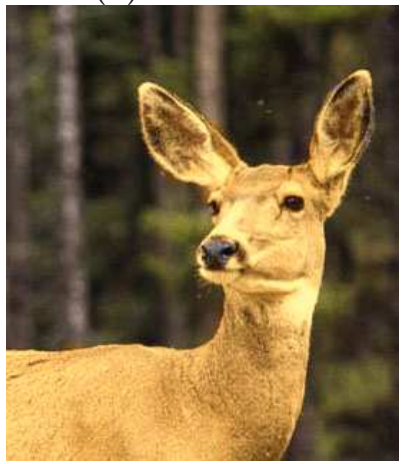

(h)

Fig. 3. Region selection using different brushes and edit propagation results, (a) original image, (b) image with strokes, (c) similar regions are selected using GMM brush without background information, (d) image with both foreground and background strokes, (e) similar regions are selected using both background and foreground information in GMM brush, (f) edit propagation based on (e) using full solution, $(\mathrm{g}$ ) result applying multi-GMM brush. (h) edit propagation based on (g) using full solution.

of its similarity with each pixel in the stroked pixel set (including both the background and foreground). Using these techniques, pixel $p$ can be efficiently classified as foreground or background by evaluating $H(p)$ : if $H(p)>\delta, p$ belongs to the foreground, otherwise $p$ belongs to background. The parameter $\delta$ can be tuned at the disposal of the users to control the amount of propagation needed.

In some situations, the user may only want to specify the foreground. To handle this case, we set $\alpha=1$ and $\beta=0$ in equation 1. If $\omega_{F}(p)$ satisfies $\left|\omega_{F}(p)-\mu_{F}\right|<\sigma$, it is considered be similar to user specified regions. An example is shown in Fig.2, our GMM brush using only foreground perfectly selects the sky region in the image (Fig. 2(b)). The GMM brush also supports selection of disconnected similar texture pattern (Fig. 2(e)). The luminance brush using luminance channel feature with a threshold [2] cannot handle texture regions that exhibit high color or luminance variation. In their approach a low threshold usually will not be able to propagate user edits to these regions, but a high threshold may propagate edits to many irrelevant regions.

The GMM brush has shown to be effective in selecting similar regions. In Fig.3, using the GMM brush with background strokes, the foreground is better detected than GMM brush without background information. Fig.9 gives another example to show the difference for GMM brushes with and without background. We also present comparison results with Li et al. [8] in Fig.9 and Fig.10 and find that GMM brush is a more effective tool to propagate user edits to similar regions.

Multiscale-GMM brush: The noise of an image will cause problems in similar region selection. For example in some images, this will make some little scattered regions to be selected incorrectly. To make the GMM selection more robust, we build the GMM models on the coarser image with detail layers attenuated. Since progressively coarser image levels increases the degree of abstraction in the resulting image [24], we build GMM for each level and combine them in a spatially varying manner to provide a more consistent similar region selection. Based on this observation, we propose a multiscale-GMM brush for similarity region selection.

Let $I$ be the input image for which we would like to construct $M$ progressively coarser images. Utilizing an edge-preserving filter, for example bilateral filtering (BLF) [25], [26] or WLS [24], we compute the progressively coarser version $I_{1}, \ldots, I_{M}$ of $I: I_{j}=$ $\frac{1}{k_{p}} \sum_{q \in N_{p}} g_{\sigma_{s}}(\|p-q\|) g_{\sigma_{r}}\left(\left\|I_{j-1}^{p}-I_{j-1}^{q}\right\|\right) I_{j-1}^{q}$, the $k_{p}=$ $\sum_{q \in N_{p}} g_{\sigma_{s}}(\|p-q\|) g_{\sigma_{r}}\left(\left\|I_{j-1}^{p}-I_{j-1}^{q}\right\|\right.$ is a normalization factor, where $g_{\sigma_{s}}$ is the spatial filter kernel and $g_{\sigma_{r}}$ is the range filter kernel. Then we build a GMM model for 
each coarser image $I_{j}$ on the user specified regions, and define MultiGMM model as the weighed sum of GMMs built from different levels:

$$
\operatorname{MultiGMM}(I)=\frac{\sum_{j=1}^{j=M} W_{j} \cdot G M M\left(I_{j}\right)}{\sum_{j=1}^{j=M} W_{j}},
$$

where the weight $W_{i}$ is defined as $g_{\sigma} * e^{2^{j} G M M\left(I_{j}\right)}$. the Gaussian convolution $g_{\sigma}$ is used to locally smooth the weight. The weight prefers coarser image by giving them larger weight because the color and luminance noise are smoothed more. From our experiments, we find out that by setting $M=4$ generates good results. We apply the bilateral grid methods [21] to accelerate bilateral filtering computing, and the progressing coarser images can be computed in real time.

We build MultiGMM models for both background and foreground: MultiGMM-B and MultiGMM-F for similar region selection. For each pixel $p$ in image $I$, we compute $\operatorname{MultiGMM-B}(p)$ and MultiGMM-F $(p)$, and then decide which part the pixel $p$ belongs to using GMM brush.

As shown in Fig. 3, Fig. 9, Fig. 10, using the MultiGMM brush, the foreground is better detected from the background. Compared with GMM brush, MultiGMM brush has the following advantages such as achieving more consistent and cleaner results, eliminating the appearance noise interfering. Fig. 3 and Fig.10 also give the edit propagation results based on our similar region selection.

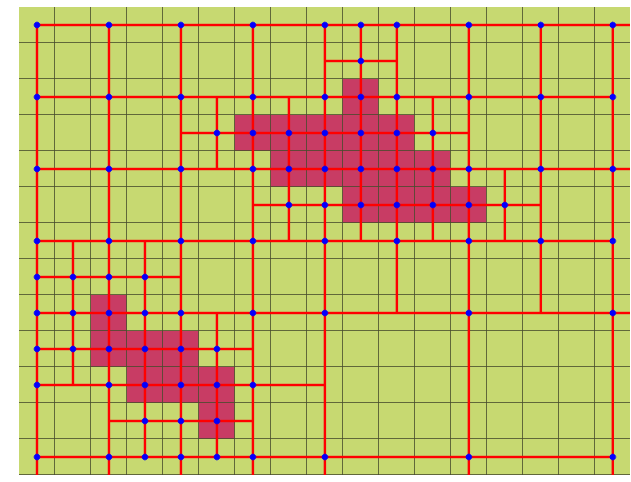

Fig. 4. Adaptive quadtree constructed from an image based on the similarity field, the red regions are the similar regions. High resolution is set on the similar regions, and coarser solution (variables) is computed on appearance different regions. Note that the variables(blue dots) are set at the center of the pixels (smallest squares).

\subsection{Constrained propagation}

Edit propagation via optimization was originally introduced by [2] for stroke-based tonal adjustment. Let $f$ be a scalar function that specifies how the appearance of each pixel needs to be adjusted, and $e(x)$ be target appearance values at the constrained pixels. The user provided constraints can be interpolated to the entire image while preserving edges of the underlying image.
To achieve this goal, inspired by [2], [4], we minimize the following quadratic function:

$$
\sum_{i} \omega_{i}\left(f_{i}-e_{i}\right)^{2}+\sum_{i}\left(\lambda+\omega_{i}\right) \sum_{j \in N_{i}} z_{i, j}\left(f_{i}-f_{j}\right)^{2}
$$

with $z_{i, j}=\exp \left(-\left\|I_{i}-I_{j}\right\|^{2} / \sigma_{t}\right) \exp \left(-\left\|x_{i}-x_{j}\right\|^{2} / \sigma_{s}\right)$, where $z_{i, j}$ is the affinity between pixel $i$ and its neighbor pixel $j, N_{i}$ is the neighborhood of pixel $p_{i}$. The weight function $\omega(x)$ indicates which and how each pixel is constrained and it is defined as follow:

$$
\omega_{i}= \begin{cases}1 & x_{i} \in \text { pixels under the foreground strokes } \\ \omega\left(x_{i}\right) & \left|\omega\left(x_{i}\right)-\mu\right|<\sigma \\ 0 & \text { otherwise }\end{cases}
$$

The first term of this energy function is a data term, responsible for satisfying the user-specified constraints. The second term is a smoothing term which keeps the gradients of the function $f$ as small as possible. We introduce an adaptive relative weight $\lambda+\omega_{i}$, which controls the relative weight of them. In our implementation the constant $\lambda$ is set to the default value $\lambda=0.1$. Using the adaptive weight, the appearance propagation have less influence on the appearance different from the stroked foreground regions. This has been proved capable of avoiding the undesirable edit propagation (Fig. 6).

The unique minimum of equation (3) is obtained by solving the following sparse linear system

$$
A f=b
$$

where $f$ is a vector with length of $n$ (the number of pixels in the image), only spatial neighbors are considered in the smoothing term, $b_{i}=\omega_{i} g_{i}$. The matrix $A$ has at most two non-zero elements per row. Since this method has to solve a large linear system, it is not scalable to multimegapixel digital imagery, for both the computational complexity and memory requirement. If we perform edit on high resolution video, the running time and space may makes it intractable on a commodity PC. This poor scalability will limit their adoption in digital photography software. Inspired by methods in [5], [6], we use the adaptively varying resolution to efficiently address this problem.

\subsection{Quadtree data structure}

According to the energy function (3), the constraints have little effects on those regions whose appearance are very different from user specified regions. The direct solution for equation (3) which has one equation for each pixel in a smooth area is unnecessary [2]. The solution in these areas can be accurately approximated by interpolating with fewer variables for each supporting a region.

Let $f_{0}$ be the original appearance of the image, the residual $b-A f_{0}$ will be zero or small for any pixel not similar to the specified regions. If we substitute $f$ with the sum $f=f_{0}+f_{\delta}$, where $f_{\delta}$ is the difference between the initial condition and final solution, the equation (4) becomes

$$
A f_{\delta}=\left(b-A f_{0}\right)
$$




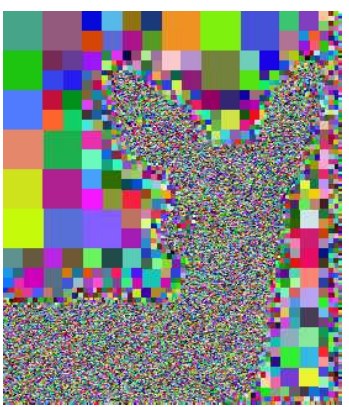

(a)

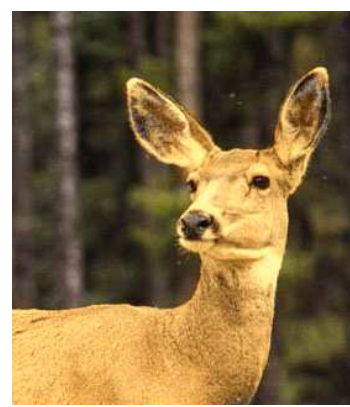

(b)

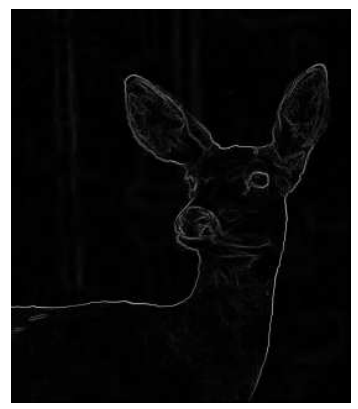

(c)

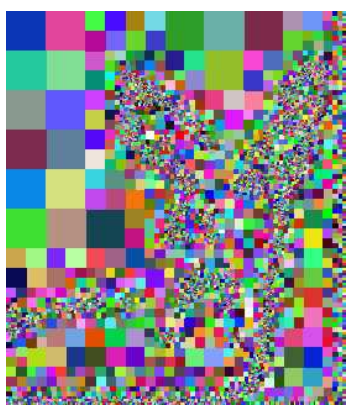

(d)

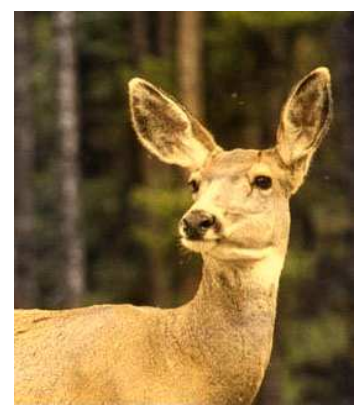

(e)

Fig. 5. Edit propagation on the reduced image, (a) Quadtree constructed on Fig. 3(g) according to the GMM similarity field, (b) edit propagation result on (a), (c) gradient of base image of Fig. 3, (d) CQD constructed on Fig. 3(g) incorporating (c), (e) edit propagation result on (d).

Note that the difference $f_{\delta}$ will be smooth and small for the regions whose appearance is very different from the specified regions. Based on this observation, we vary the resolution of a solution vector adaptively according to a similarity field. High resolution is used at the regions that are most similar to the user specified ones, and progressively lower resolution in areas whose appearance is less similar.

To compute the adaptive subdivision, inspired by the algorithm [5], we decompose the entire image (a vector of dimension $n$ ) hierarchically using a quadtree data structure. Using this method, we obtain a set of sparse and adaptive samples $y$ of the original image (y is a vector of dimension $m$, usually $m<<n$ ). The linear system formed by the samples $y$ is called the reduced problem. The appearance on $y$ is defined as $g$. By substituting $f=U g$, the full resolution problem in equation (3) is then transformed into a smaller linear system defined on a reduced space

$$
A U g=b
$$

where $U$ is an $m \times n$ matrix that transforms from the reduced space to the full space. The normal equations for equation (5) is

$$
U^{T} A^{T} A U g_{\delta}=U^{T} A^{T}\left(b-A U g_{0}\right)
$$

With the matrix $U^{T} A^{T} A U$ and vector $U^{T} A^{T}(b-$ $\left.A U g_{0}\right)$ are computed, this linear system becomes an $m \times m$ sparse matrix-vector multiplication, which can be solved using preconditioned conjugate gradients [27] or the locally adapted hierarchical basis preconditioning (LHB) [19].

To efficiently define the sample set $y$, the similarity measure $w(p)$ is used to build the quadtree data structure. The larger $w(p)$, the more similar the pixel $p$ is to the user specified region. We adaptively subdivide the space using a quadtree [28] that is maximally subdivided to pixel level nodes in the similarity regions (Fig. 4). Firstly, we label the pixels according to the following measurement metric: If the weight value $w(p)$ of all pixels are between $[a, b]$, assuming we would sort the pixels into $\mathrm{S}$ clusters. The pixels are label $L(p)=i$ if $w(p) \in[a+i *(b-a) / S, a+(i+1) *(b-a) / S]$, using this simple and efficient scheme, we efficiently cluster the pixels in the entire image (Fig. 4).

Based on the computed pixel label $L(p)$ and the quadtree construction method [28], we can build the quadtree with a pointer-based tree structure. The quadtree is subdivided based on two criteria: one is the labels of the pixels in the node, if the number of the labels is above a given threshold, this node is further subdivided; the other criterion is to set the pixel located in the similar region to be finest resolution. Similar to [5], variables in the reduced space (elements of $g$ ) are placed at the corners between leaf nodes, as shown in Fig. 4(b). Each leaf node stores the indices of the variables at its four corners. Given this quadtree and the values of a vector $g_{\delta}$, we apply the four neighbor quadtree traversal algorithm [29] to generate the reduced linear system (6). Note that the matrix $S$ does not need to be explicitly built since it simply encodes a bi-linear interpolation from quadtree nodes to pixels. The interpolation $f_{\delta}=U g_{\delta}$ can be computed with a single traversal of the quadtree. The final tonal adjustment result of the image is $f=f_{0}+f_{\delta}$.

We call this method partial quadtree decomposition (PQD) since we represent each pixel on the similar regions with a single variable. As illustrated in Fig. 1, Fig. 6, Fig. 9 and Fig. 11, we achieve visually similar result to the full solution. Note that in Fig 6, the provided adaptive relative weight efficiently prevents undesirable edit propagation.

\subsection{Quadtree constructed in the similar regions}

There are usually situations when regions that are similar to the user selection occupy a large part of the image. An example is shown in Fig. 1, Fig. 5, Fig. 6. In this case, using the Partial Quadtree Decomposition (PQD) may not be efficient enough. We further construct an adaptive quadtree in the similar regions. But different from PQD, since the appearance are usually very similar in these regions, instead of constructing the quadtree based on similarly measure, we utilize the edge information of the image to guide the adaptive subdivision on the similar 


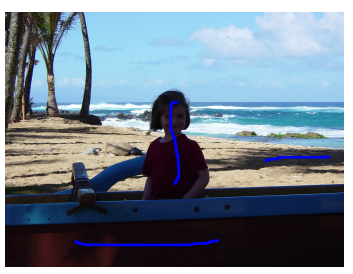

(a)

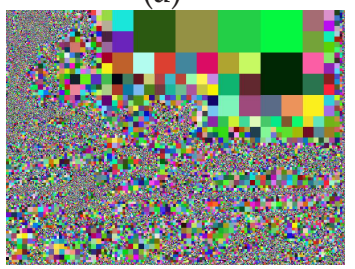

(f)

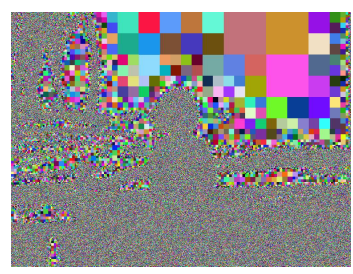

(b)

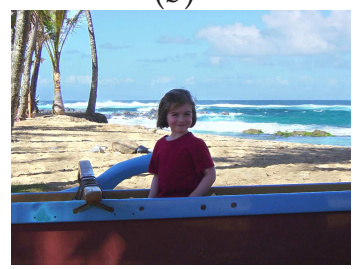

(g)

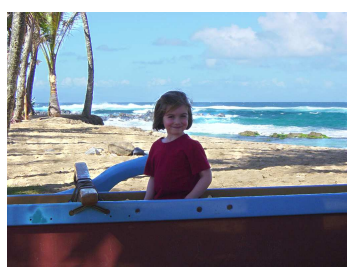

(c)

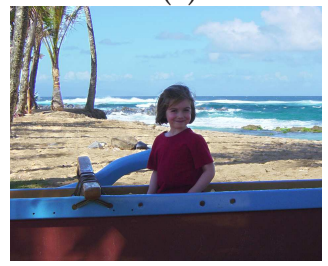

(h)

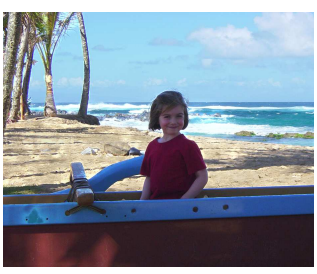

(d)

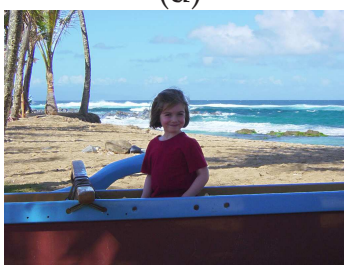

(i)

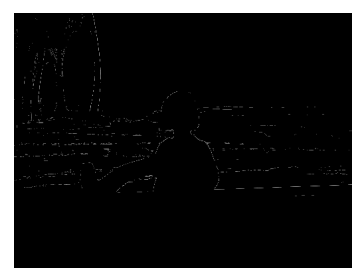

(e)

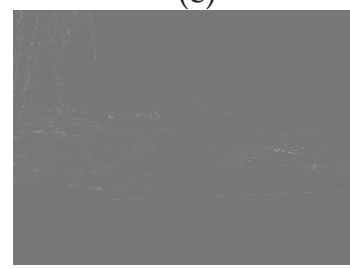

(j)

Fig. 6. Appearance propagation in ordinary low dynamic range image. (a) The strokes on the image, (b) Quadtree constructed on (a) according to the GMM similarity field, (c) accelerated solution using PQD, (d) accelerated solution using PQD with adaptive relative weight, (e) extracted edge information, $(f)$ complete quadtree constructed on (a) based on similarity field and edge information, (g) accelerated solution using CQD, (h) accelerated solution using CQD with adaptive relative weight, (i) full solution, (j) the error visualization between CQD result (h) and full solution result (i).

regions. To avoid direct image edge detection, which is a well known difficult problem, we use the derivative of the base image of [30] to guide the quadtree construction. We call this method Complete Quadtree Decomposition (CQD).

We apply the bilateral filter [25], [26] to create edgepreserving base images from the original image. More formally, let $I$ be the original image, and $I_{g}$ be the grayscale (intensity) image of $I$, filtering $I_{g}$ using the bilateral filter results in a large-scale layer $I_{B}$. As the base image $I_{B}$ preserves strong edges and small changes in intensity are smoothed out. To detect the prominent edges of the image, we evaluate the derivative of the base image $\nabla\left(I_{B}\right)$. Given a threshold $\sigma$, if amplitude $\left|\nabla\left(I_{B}\right)\right|$ is greater than $\sigma$, it is considered as the an edge. As illustrated in Fig. 6, using this approach, the edge information is efficiently detected. By applying bilateral grid techniques [21], the prominent edges can be extracted in real time.

We also incorporate the information of the multiscale progressively coarser images. First progressively coarser version $I_{1}, \ldots, I_{M}$ of grayscale of $I_{g}$ is computed, then we calculate the derivative $\nabla\left(I_{j}\right)$ for each coarser image $I_{j}$, and define multiscale edge detection model as the weighed sum of image derivative in the $M$ image sequences:

$$
\operatorname{MultiD}\left(I_{g}\right)=\frac{\sum_{j=1}^{j=M} w_{j} \cdot\left|\nabla\left(I_{j}\right)\right|}{\sum_{j=1}^{j=M} w_{j}},
$$

where the weight $w_{i}$ is defined as $g_{\sigma} * e^{2^{j}}\left|\nabla\left(I_{j}\right)\right|$, similar to the Multi-GMM brush, the weight prefers coarser image. We then detect the edge by evaluating $\operatorname{MultiD}\left(I_{g}\right)$. We set $M=3$ in all our examples and generate good results.

The prominent edges of the image is used to guide the adaptive quadtree construction in similar regions. The maximum subdivision occurs along the maximum edges of the base image. To avoid oversampling, we set some threshold $\sigma$ for the edge (measure for edge strength). Only those pixels above a threshold (specified by the user) are considered as an edge. Compared with PQD, CQD method uses a hybrid decomposition method which makes the image further subdivided on the similar regions, and generate a much smaller space.

Note that when edge information is weak (under a user given threshold) in the appearance similar regions, such as Fig. 1 and Fig. 5, the similar regions can be sampled without considering the edge information. When there are strong edge information in the appearance similar regions such as Fig. 6, we put more samples on the edge regions.

The CQD method is extremely efficient even when processing the appearance propagation with large similar regions. This is shown in Fig. 1, Fig. 5 and Fig. 6 . For example, for a $720 \times 640$ image (Fig.1), it takes approximate $1.8 \mathrm{~s}$ total using the PQD method, while it take only $0.1 \mathrm{~s}$ using the PQD method and no obvious difference can be observed between the results. More CQD results are shown in Fig. 11 and Fig.13.

In CQD method, the adaptive relative weight in equation 3 is effective in preventing undesirable edit propagation. Fig. 6 gives the experimental comparison results with and without adaptive relative weight in CQD method. The visualizations of error between our CQD method and full solution (Fig. 1, Fig. 6 and Fig. 11) further convince our conclusion.

\section{VIDEO EDIT PROPAGATION}

In this section we apply the hierarchical data structure to video data and present an efficient video edit propa- 
gation algorithm. Video edit propagation is achieved by minimizing the following quadratic function:

$$
\begin{aligned}
f= & \arg \min _{f}\left\{\sum_{i} \omega_{i}\left(f_{i}-g_{i}\right)^{2}+\alpha \sum_{i} \sum_{j \in N_{s i}} z_{S_{i, j}}\left(f_{i}-f_{j}\right)^{2}\right. \\
& \left.+\beta \sum_{i} \sum_{j \in N_{T i}} z_{T_{i, j}}\left(f_{i}-f_{j}\right)^{2}\right\}
\end{aligned}
$$

with $z_{i, j}=\exp \left(-\left\|I_{i}-I_{j}\right\|^{2} / \sigma_{t}\right) \exp \left(-\left\|x_{i}-x_{j}\right\|^{2} / \sigma_{s}\right)$, where $N_{S i}=N_{S 4}(i)$ is the 4 spatial neighborhood of pixel $i$, and $N_{T i}=N_{T 2}(i)$ is pixel $i^{\prime}$ 's 2 temporal neighborhood. The first term is a data term, measuring the conformity of the user specified non-overlapping constraints. The second term is a smoothing term, which preserves the gradients of the function $f$ between adjacent pixels as small as possible. The third term measures smoothness of exposure function between two adjacent pixels in two adjacent frames. By minimizing the above energy function, we can derive the following similar linear system:

$$
A^{*} f=b^{*}
$$

where

$$
\begin{aligned}
A_{i, j}^{*} & = \begin{cases}-\alpha z_{S i j} & j \in N_{S}(i) \\
-\beta z_{T i j} & j \in N_{T}(i) \\
w(i)-\sum_{k \in N_{S i}} A_{i, k}^{*}-\sum_{k \in N_{T i}} A_{i, k}^{*} & (i=j) \\
0 & \text { otherwise }\end{cases} \\
b^{*}(i) & =w(i) g(i)
\end{aligned}
$$

For video data, equation (10) is usually a very large linear system. Even short video sequences contain a large number of pixels (e.g.,100M pixels in a 10 second shot), the scale of the problem makes it computationally infeasible to run edit propagation at the pixel level. To solve this problem, similar to the image edit propagation acceleration case, we propose an adaptive hierarchical octree data structure. This octree is generated by the similarity measure on the video which is computed using GMM brush. Then the propagation is done in the reduced space, and the full resolution results are obtained by interpolating the derived solution. In the case when the size of similar regions is large compared with the entire video, we further subdivide the similar regions guided by the edge surface information. We use the method proposed in [21] to efficiently extract the large-scale parts of original video. To indicate affected objects in the video, we can apply the user interface presented in [31] by painting on arbitrary spatio-temporal surfaces in the video volume.

We apply the eight neighbor quadtree traversal algorithm of [29] for the setup of the reduced linear system. Then we define the energy function (9) in this significantly reduced space. Within this reduced space, the interactive appearance adjustment can be done very efficiently and provides instant feedback to users as they paint new strokes in the video volume. Our method efficiently addresses the computation problem of edit propagation for video, as shown in Fig. 7, 8, 14. The appearance propagations are performed efficiently and satisfying results are obtained.

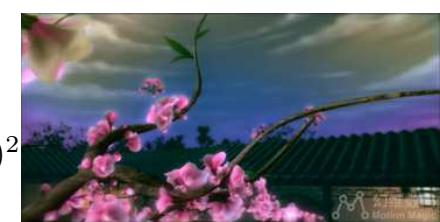

(a)

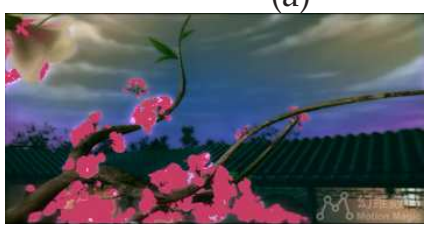

(c)

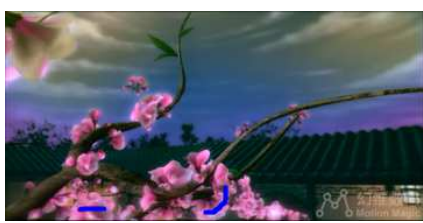

(b)

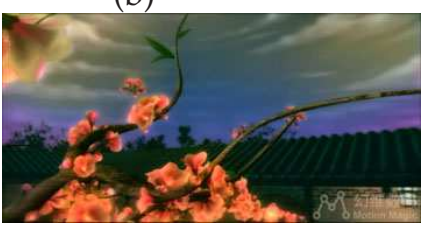

(d)
Fig. 7. Appearance propagation in video $(1080 \times 480 \times 46)$. (a) original video (the 1th frame), (b) the stroke on the video, (c) the selected similar region, (d) the result of tonal adjustment.

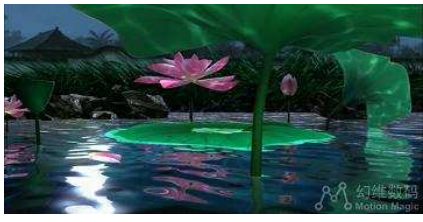

(a)

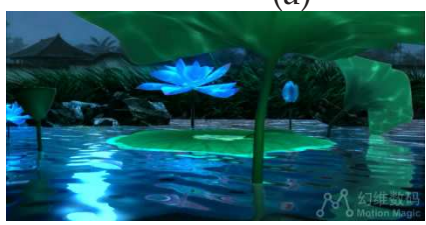

(c)

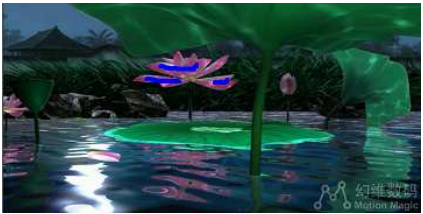

(b)

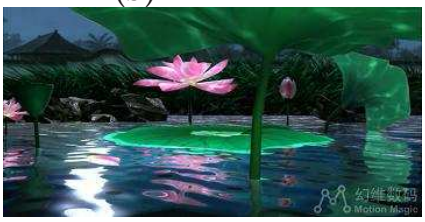

(d)
Fig. 8. Appearance propagation in video $(720 \times 360 \times 75)$. (a) original video (the 1th frame), (b) the stroke on the video, (c) result after tonal adjustment, (d) another result.

\section{COMPUTATIONAL COMPLEXITY ANALYSIS}

The quadtree complexity theorem [32] states that the size of the quadtree representation of a region is linear in the perimeter of the region. Meagher [33] states that the Quadtree complexity theorem also holds for three dimensional data (octree instead of quadtree) where perimeter is replaced by surface area as well as higher dimensions: the size of the $k$-dimensional quadtree of a $k$-dimensional object is proportional to the sum of the resolution of the quadtree and the size of the $(k-1)$ dimensional exterior interface of the object. This means that the application of a quadtree algorithm to a problem in $d$-dimensional space executes in time proportional to the array-based algorithm in the $(d-1)$-dimensional space of the original $d$ dimensional image. Algorithms that execute on a quadtree representation of an image have an execution time that is proportional to the number of blocks in the image rather than the number of pixels, the memory requirement is reduced accordingly.

Since our edit propagation function is constructed using the hierarchical data structure (quadtree and octree), both the memory consumption and time complexity 


\begin{tabular}{|c|c|c|c|c|c|}
\hline Data & Mpixels & $\operatorname{Vars}(\%)$ & $\begin{array}{l}\text { Error } \\
\text { RMS Max }\end{array}$ & $\begin{array}{l}\text { Times(s) } \\
\text { QT LHB }\end{array}$ & $\begin{array}{l}\text { Memory } \\
\text { QT LHB }\end{array}$ \\
\hline Sky & 0.15 & 0.49 & $0.0146 \quad 1.3$ & $\begin{array}{ll}0.1 & 1.7\end{array}$ & 254 \\
\hline Swan & 0.82 & 0.94 & 0.01020 .36 & $0.94 \quad 13$ & $8 \quad 115$ \\
\hline Girl & 5.82 & 0.32 & $0.0148 \quad 1.7$ & $1.25 \quad 35$ & $14 \quad 345$ \\
\hline
\end{tabular}

TABLE 1

Performance comparison and error value between our result and full solution.

\begin{tabular}{|lll|cc|}
\hline Data & Mpixels & Vars(\%) & Times(s) & Memory(MB) \\
\hline Waterlily & 20 & 0.18 & 11 & 46 \\
Flower & 24 & 0.45 & 12 & 72 \\
Waterfall & 12 & 0.15 & 6 & 40 \\
boom & 12 & 0.12 & 8 & 58 \\
\hline
\end{tabular}

TABLE 2

Performance of octree-based edit propagation for videos.

are significantly reduced. They are bounded by the solution of the reduced $m$-variable linear system (7). The Quadtree complexity theorem [32] shows that the number of quadtree nodes in an image quadtree is $O(p)$, where $p$ is the total length of the prominent edges (which is measured with a threshold $\sigma$ ) in the image if using the CQD method. Since $m$ is linearly proportional to the number of leaf nodes in the quadtree, $m$ is $O(p)$. The growth of $p$ will depend on how the edges are chosen on the image, for typical cases, we observe that $p$ is $O(\sqrt{n})$. In case of video volume, in our algorithm, the octree is densely sampled around the boundary surface, the number of octree nodes in a volume octree is $O(p)$, where $p$ is the total area of the boundary surfaces. We use the top-down pointer-based quadtree traversal algorithm [29] for 4-neighbor traversal (also 8-neighbor traversal), the representation requires as few as $2 N$ bytes, where $N$ is the number of nodes in the tree.

\section{Results AND discussion}

In order to show the effectiveness of the proposed approach, we test it using different datasets. The examples presented are different type of data including ordinary LDR images, HDR images, and high-resolution videos. Performance of these algorithms are measured on an Intel Core 2 Duo $2.6 \mathrm{GHz}$ machine with 2GB RAM.

In Fig.9 and Fig.10, we compare our brushes with the scribbleboost classifier [8] and lazy snapping [9]. It should be pointed out that we implement both our methods and scribbleboost classifier [8] without using the post-processing step nor extra features presented in [8].Our GMM brush is defined as weighted sum of similarity values from both foreground and background. Furthermore, our multi-GMM brush is performed on the progressively smoother image, these make our methods more robust and generate more consistent and cleaner results. As illustrated in Fig.9 and Fig.10, compared with the results of [8], our GMM brush and multi-GMM brush generate much cleaner results. The occasional isolated pixels can be eliminated more easily. Lazy Snapping [9] applies graph cuts to create binary masks, which prefer to generate more closed and consistent regions, while our method tends to select all regions (may not be connected) that are similar to the stroked regions, which is more appropriate for edit propagation.

In Table 1, we give the performance comparison between our quadtree based algorithm(QT) and locally adapted hierarchical basis preconditioning (LHB) algorithm [19] for several images. The LHB preconditioning [19] is one of the fastest approaches in solving large linear system. In all the comparisons, we performed our method based on the following criteria: receiving the most visually identical result to full solution using [19] while requiring the lowest resolution to construct the quadtree. For each dataset, we show the number of the pixels, the number of variables in the reduced linear system as the percentage of the total number of pixels. We also measure the error between the solutions computed using the reduced and full linear system (error is measured using the $\mathrm{L}$ channel of CIE $L^{*} a^{*} b^{*}$ color space.) with both an average per-pixel RMS error and the maximum error across all pixels). Time and memory requirement are also compared.

We compare with [4] for both performance and speed. The running time and memory requirements of [4] are based on both the image size and the number of sampled columns of derived affinity matrix. All the comparisons are based on the same scribble constraints. As shown in Fig. 13, for a $644 \times 874$ image, our method takes about $0.1 \mathrm{~s}$ and $8 \mathrm{MB}$ memory, it takes about $4 \mathrm{~s}$ total and $96 \mathrm{MB}$ memory for [4]. In Fig. 11, for a $560 \times 400$ image, our method takes $0.3 \mathrm{~s}$ and $5 \mathrm{MB}$ memory, while it takes about $1.8 \mathrm{~s}$ and $45 \mathrm{MB}$ memory for [4]. However, as shown in these figures, our results are visually identical to [4].

We also compare our results with [22] and [23]. With our multi-GMM similarity measure, the appearance similar regions are more accurately selected. It can also be observed that in Fig.12(a) and (b), our method can tune the illuminance better (see the trees). Furthermore, using our CQD method, we build adaptive quatree in the similar regions, our method is faster. For the image $(2048 \times 1536)$ in Fig.12, our method takes $1.25 \mathrm{~s}$, while $\mathrm{Xu}$ et al. [22] takes 4.25s. The edge aware wavelets [23] are very fast, for image in Fig.12(d) with $1920 \times 1284$, the computational time is $3.54 \mathrm{~s}$, while our method takes $0.68 \mathrm{~s}$. However, when performing edit propagation on image with weak edges, this method will generate over propagation results, as shown in Fig.12(d). With the same constrains, our method avoids this artifact.

Fig. 1, Fig. 5 and Fig. 6 show that our method can efficiently edit the ordinary low dynamic range image, and the comparison results between our PQD and CQD methods and full solution are also shown. In the case when similar regions occupy a large part of the image, if we only subdivide the less similar regions (PQD method), the computational complexity and memory re- 


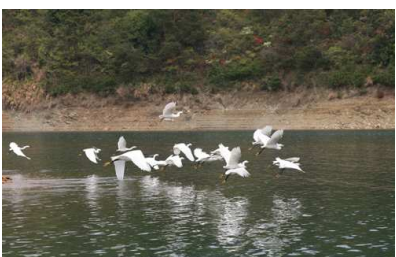

(a)

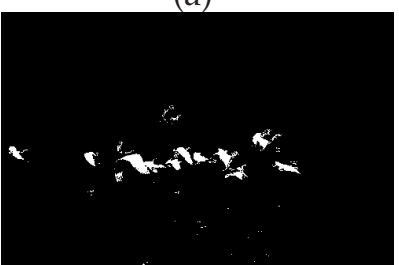

(e)

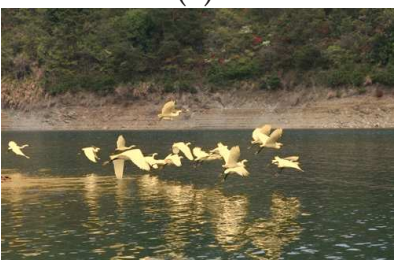

(i)

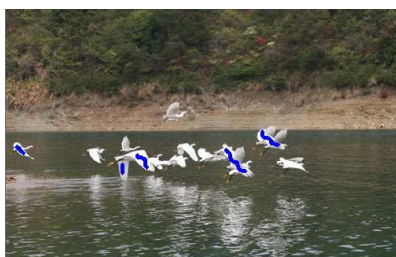

(b)

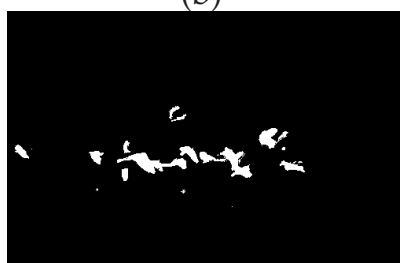

(f)

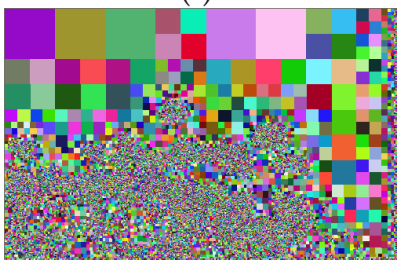

(j)

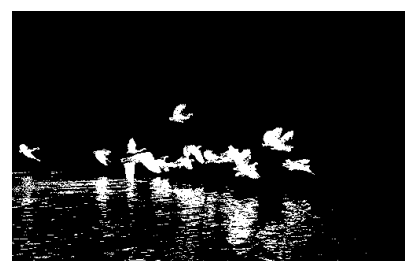

(c)

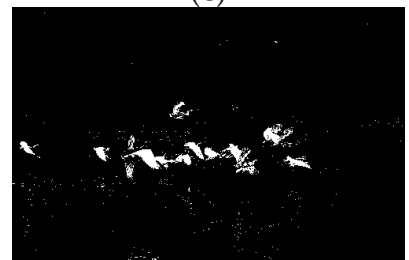

(g)

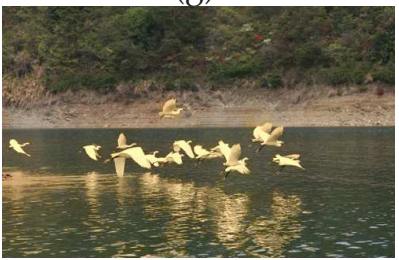

(k)

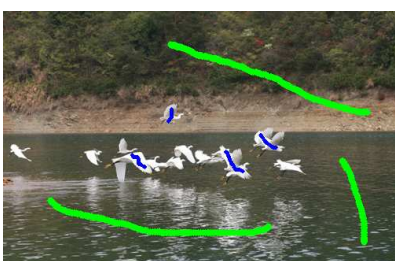

(d)

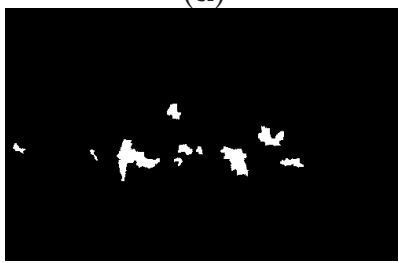

(h)

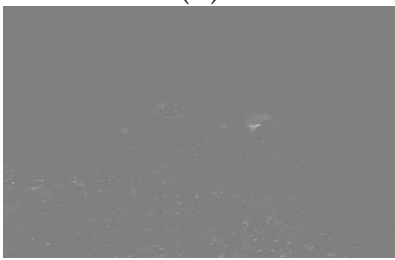

(1)

Fig. 9. (a) Original image, (b) image with strokes on the foreground, (c) similar regions are selected using GMM brush, (d) image with strokes on both the foreground and background, (e) similar regions are selected using GMM brush incorporating both the foreground and background information, (f) Multi-GMM brush, (g) ScribbleBoost [8], (h) Lazy snapping [9], (i) appearance edit on (c) using full solution, (j) adaptive quadtree built on image (c), (k) edit propagation results computed on quadtree (j), (l)the visualization of error between the solution (i) and solution (k).

quirement is still high. However, when we use the CQD method, the time and space requirement is significantly reduced. For example, in Fig. 6 it takes 0.5 seconds to complete the computation using PQD method, and it take 0.05 seconds using the CQD method. But both the results are visually identical to the full resolution solution. The error between our CQD method and full solution is also shown.

Fig. 13 shows that our method can edit high dynamic range images. We adjust several regions, such as the window, book, and the desktop, to obtain the desired brighter result. we illustrate the comparisons of different algorithms [34], [35] on the tone mapping of an HDR image (OpenEXR images). We use only simple brush strokes shown in Fig.13(c) to adjust the exposure and contrast. Compared with full resolution solution, as shown in Table 1, our algorithm is much faster, the memory requirement is significantly less, while the solution is visually identical to the full solution. In our algorithm, we apply the exrdisplay program, which is included in the OpenEXR software distribution, to display "raw" 16bit OpenEXR image on our 8-bit monitor. The exrdisplay changes the exposure but does not affect image data itself.

Fig. 8, Fig. 7 and Fig. 14 present several video edit propagation results that were too large to compute in a common PC using full resolution solution [19]. Our hierarchical data structure framework is very effective for a variety of objects in the videoes, from blooming flowers to flowing streams. For example, in Fig. 8, the tone of water lily is adjusted, and the tone of its inverted reflection is also adjusted respectively. The running time, memory consumption and other experimental data for video edit propagation examples (Fig. 8, 7, 14) are shown in Table 2. In general user interaction time depends on the complexity of the scene. Videos with complex and thin structures, fast motion may require more strokes to guide the system. In Fig. 7 and 8 , despite of the strong edges and almost constant colors of flowers in this video sequence, our approach is able to fully extract the foreground by painting only a few strokes on a single frame of the video.

\section{Limitations:}

Our framework works well for fast edit propagation. However, our methods have the following limitations. One component that can be improved is the edge detection method used to guide the adaptive quadtree and octree structure in the similar regions. Better edge detection methods could be more effective in identifying fuzzy or complex region edges. Although our GMM rush works well for selecting similar appearance regions, however, when the foreground is extremely complex, such as the object in Fig.15, our method can not select the foreground accurately. The inaccurate foreground selection may generate unpleasant results, as shown in Fig.15. To address this problem, one feasible method is to use the interactive alpha image matting techniques [10]. Another drawback is that our system requires sev- 


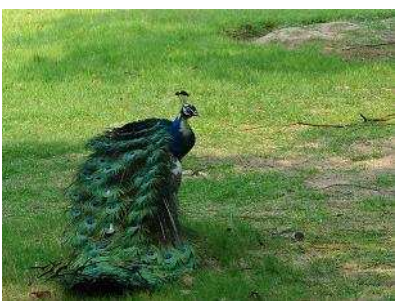

(a)

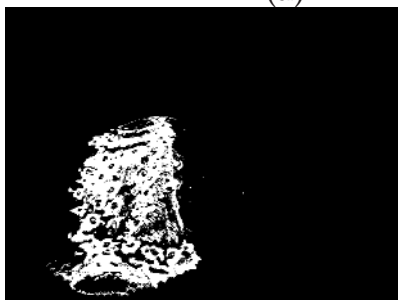

(e)

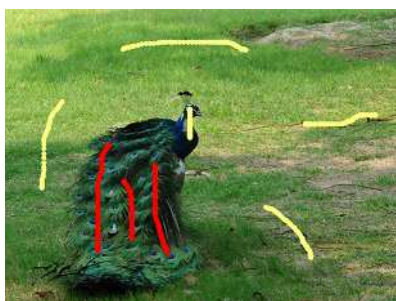

(b)

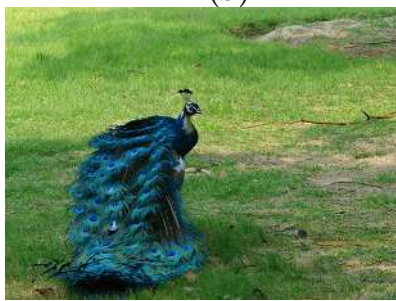

(f)

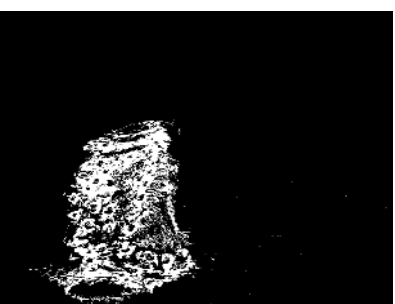

(c)

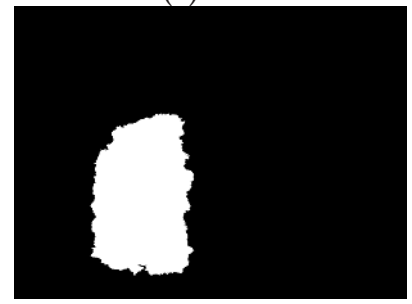

(g)

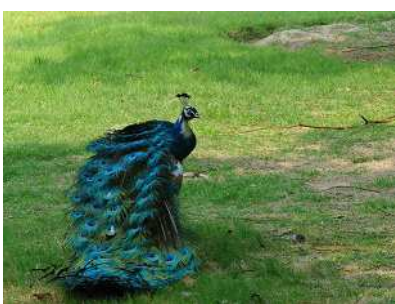

(d)

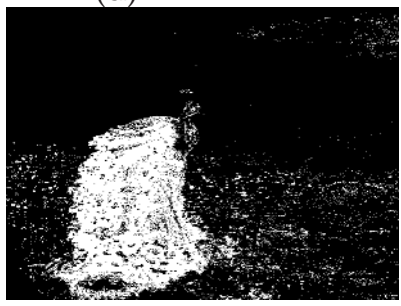

(h)

Fig. 10. (a) Original image, (b) image with strokes, (c) similar regions are selected using GMM brush, (d) edit result on (c) using full solution, (e) similar regions are selected using multi-GMM brush, (f) edit result on (e) using full solution, (g)(h) similar regions are selected using Lazy snapping [9] and ScribbleBoost [8], respectively.

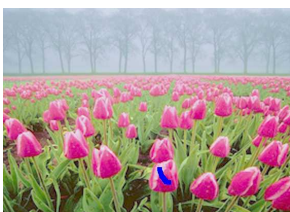

(a)

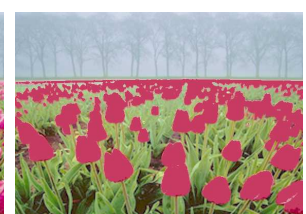

(b)

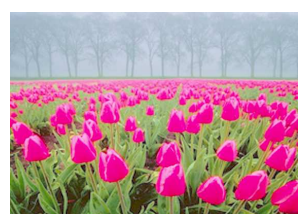

(c)

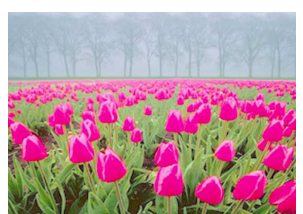

(d)

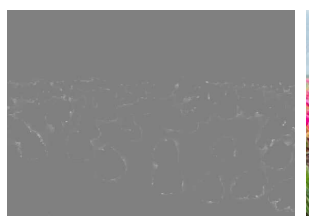

(e)

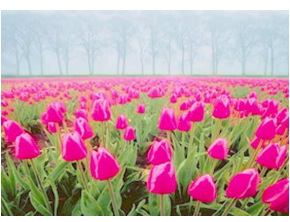

(f)

Fig. 11. Tonal adjustment of a tulip HDR image and comparison with [An and Pellacini 2008]. (a) Stoke drawn on original HDR image, (b) selected similar regions using GMM brush, (c) result generated using our CQD method. (d) result generated using full solution. (e) the visualization of error between our CQD method(c) and full solution (d). (f) result generated using with [An and Pellacini 2008]. (HDR image (C)Jaap Hart. All rights reserved).

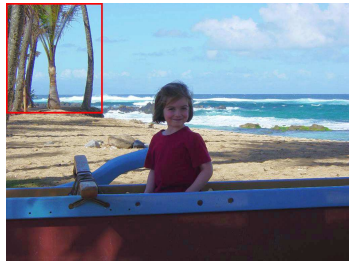

(a)

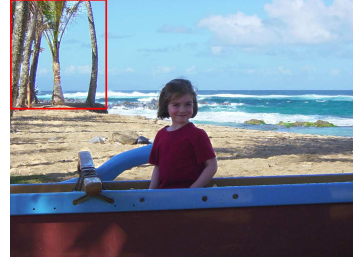

(b)

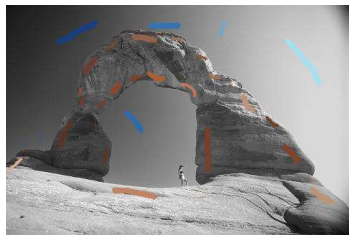

(c)

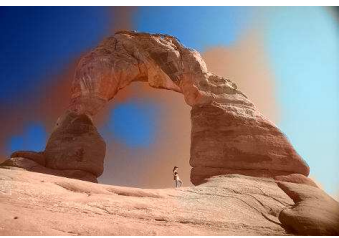

(d)

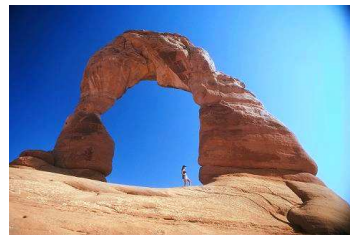

(e)

Fig. 12. Comparison with Xu et al. [22] and Fattal [23]. (a) result of Xu et al. [22], (b) result of our method, (c) Input image with srokes, (d) result of Fattal [23], (e) result of our method.

eral parameters to subdivide the image using quadtree construction, which are not intuitive to select for nonresearchers. We plan to develop a method to compute the parameters by learning from the image that is being edited by the professionals in the future.

\section{CONCLUSION AND FUTURE WORK}

This paper presents a novel hierarchical data structure to speed up edit propagation. Using the proposed approach, the large linear system in existing optimization based propagation methods [19] is reduced to a dramatically smaller linear system. This makes the time and space requirement significantly reduced, while the result is almost visually identical to the full resolution solution. Our method can effectively solve the scalability problem of appearance edit propagation in photography. We demonstrate the effectiveness of our edit propagation framework on both images and videos.

In the future, we are interested in investigating the following topics: first, we would like to apply our method to process other types of visual data, such as measured materials, and volumetric datasets; second, we would like to construct a more accurate octree structure for video, we would incorporate optical flow techniques in our framework; third, we will extend our approach for interactive image and video enhancement [36] and 


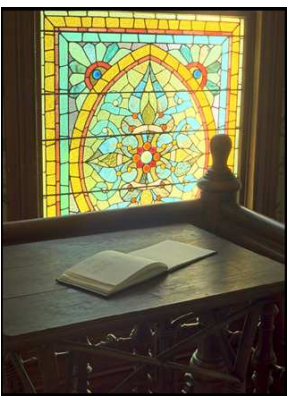

(a)

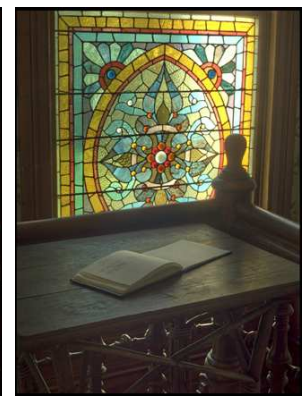

(b)

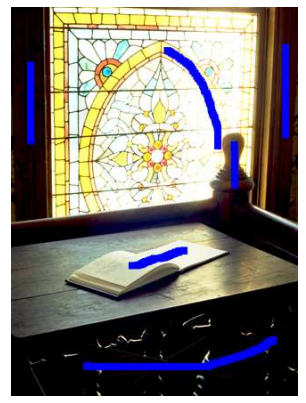

(c)

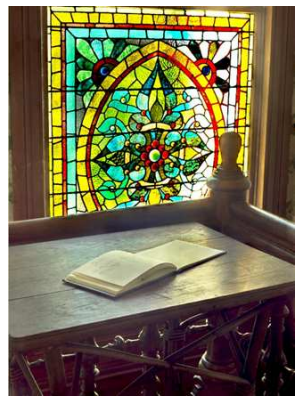

(d)

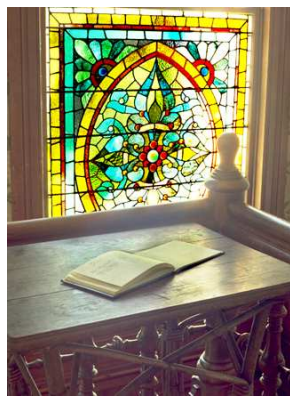

(e)

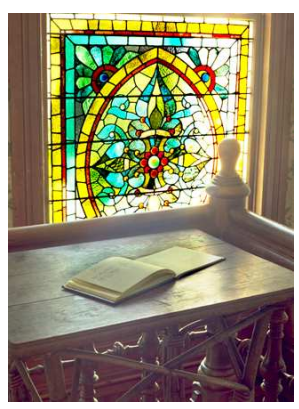

(f)

Fig. 13. (a) Tone mapping produced by the gradient domain method of Fattal et al.[2002], (b) tone mapping produced by Reinhard's operator [2002], (c) original HDR image with strokes, (d) the result using full solution, (e) the result produced using our method, (f) the result produced using [An and Pellacini 2008] (HDR image c Industrial Light \& Magic. All rights reserved.)

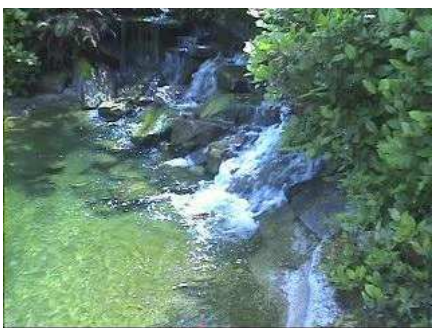

(a)

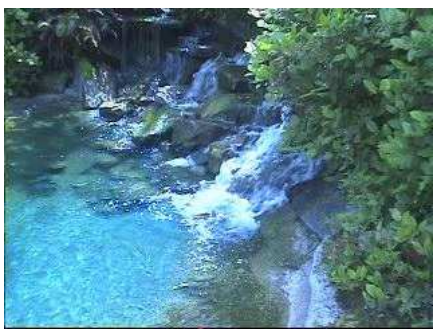

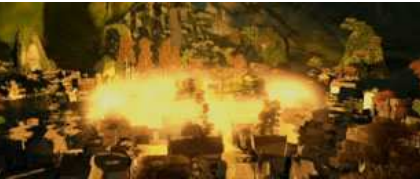

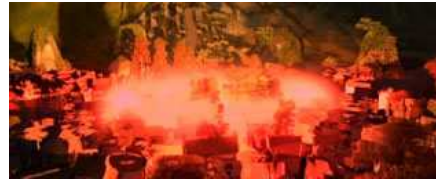

(b)

Fig. 14. (a) Video edit propagation, left: the 1th frame of the original video $(640 \times 496 \times 150)$, right: the edited result. (b) Video edit propagation, left: the 1 th frame of the original video $(624 \times 256 \times 70)$, right: the edited result.

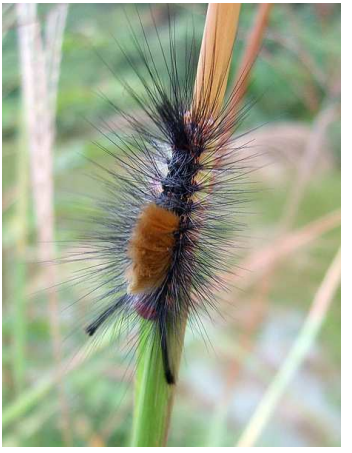

(a)

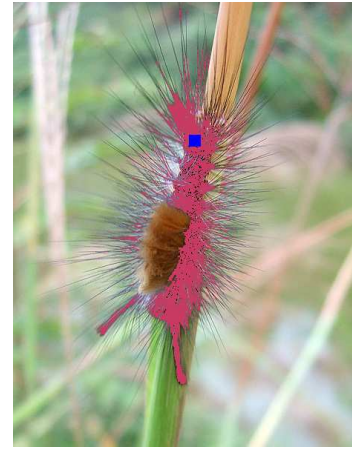

(b)

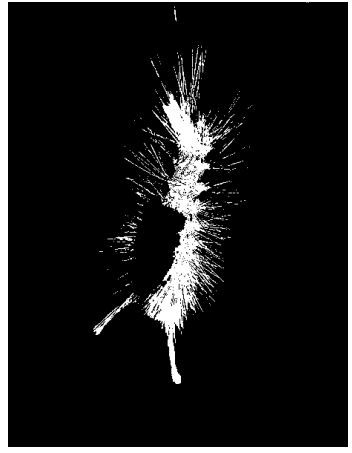

(c)

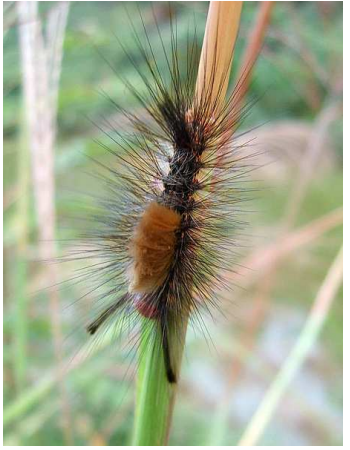

(d)

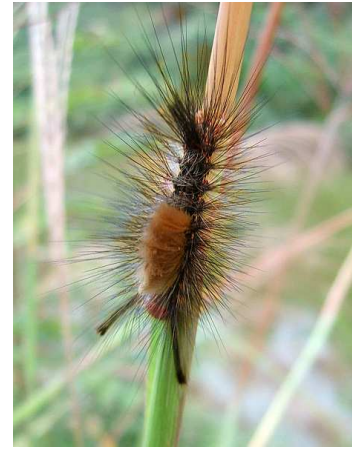

(e)

Fig. 15. Failure example. (a) Original image, (b) the stroke on the foreground and selected similar appearance regions using GMM brush, (c) masked image of the selected foreground, (d) image edit propagation result, (e) image edit propagation result using different tone parameter.

perform image detail enhancement propagation.

\section{ACKNOWLEDGMENT}

We would like to thank the anonymous reviewers for their valuable comments and insightful suggestions. This work was partly supported by NSFC (No. 60803081), National High Technology Research and Development Program of China (863 Program) (No. 2008AA121603), Natural Science Foundation of Hubei Province (2008CDB350), State Key Lab of CAD\&CG (No. A0808), State Key Laboratory of Software Engineering (SKLSE) (No. SKLSE2008-07- 08).

\section{REFERENCES}

[1] INC. ADOBE SYSTEMS. Adobe Photoshop CS2. In Adobe Systems, Inc., San Jose, CA, 2005.

[2] D. Lischinski, Z. Farbman, M. Uyttendaele, and R. Szeliski. Interactive local adjustment of tonal values. Proceedings of $A C M$ SIGGRAPH 2006, 25(3):646-653, 2006.

[3] F. Pellacini and J. Lawrence. AppWand: editing measured materials using appearance-driven optimization. In SIGGRAPH. ACM Press New York, NY, USA, 2007.

[4] X. An and F. Pellacini. AppProp: all-pairs appearance-space edit propagation. In Proceedings of ACM SIGGRAPH 2008. ACM New York, NY, USA, 2008.

[5] A. Agarwala. Efficient Gradient-Domain Compositing Using Quadtrees. ACM TRANSACTIONS ON GRAPHICS, 26(3):94, 2007.

[6] F. Losasso, F. Gibou, and R. Fedkiw. Simulating water and smoke 
with an octree data structure. ACM Transactions on Graphics (TOG), 23(3):457-462, 2004.

[7] A. Levin, D. Lischinski, and Y. Weiss. Colorization using optimization. ACM Transactions on Graphics (TOG), 23(3):689-694, 2004.

[8] Y. Li, E. Adelson, and A. Agarwala. ScribbleBoost: Adding Classification to Edge-Aware Interpolation of Local Image and Video Adjustments. In Computer Graphics Forum, volume 27, pages 1255-1264. Blackwell Publishing Ltd, 2008.

[9] Y. Li, J. Sun, C.K. Tang, and H.Y. Shum. Lazy snapping. In SIGGRAPH, pages 303-308. ACM Press New York, NY, USA, 2004.

[10] J. Wang and MF Cohen. An Iterative Optimization Approach for Unified Image Segmentation and Matting. In ICCV 2005, volume 2, 2005.

[11] Y. Qu, T.T. Wong, and P.A. Heng. Manga colorization. Proceedings of ACM SIGGRAPH 2006, 25(3):1214-1220, 2006.

[12] Q. Luan, F. Wen, D. Cohen-Or, L. Liang, Y.Q. Xu, and H.Y. Shum. Natural image colorization. In Rendering Techniques 2007 (Proceedings Eurographics Symposium on Rendering). Citeseer, 2007.

[13] L. Yatziv and G. Sapiro. Fast image and video colorization using chrominance blending. IEEE Transactions on Image Processing, 15(5):1120-1129, 2006.

[14] C. Xiao, S. Liu, H. Fu, C. Lin, C. Song, Z. Huang, F. He, and Q. Peng. Video completion and synthesis. Computer Animation and Virtual Worlds, 19(3-4):341-353, 2008.

[15] C. Rother, V. Kolmogorov, and A. Blake. " GrabCut": interactive foreground extraction using iterated graph cuts. ACM Transactions on Graphics (TOG), 23(3):309-314, 2004.

[16] X. Bai and G. Sapiro. A geodesic framework for fast interactive image and video segmentation and matting. In ICCV, pages $1-8$, 2007.

[17] Y. Saad. Iterative Methods for Sparse Linear Systems. Society for Industrial Mathematics, 2003.

[18] J. Bolz, I. Farmer, E. Grinspun, and P. Schröoder. Sparse matrix solvers on the GPU: conjugate gradients and multigrid. In SIGGRAPH, pages 917-924. ACM New York, NY, USA, 2003.

[19] R. Szeliski. Locally adapted hierarchical basis preconditioning. In SIGGRAPH, pages 1135-1143. ACM New York, NY, USA, 2006.

[20] R. Szeliski and H.Y. Shum. Motion estimation with quadtree splines. Pattern Analysis and Machine Intelligence, IEEE Transactions on, 18(12):1199-1210, 1996.

[21] J. Chen, S. Paris, and F. Durand. Real-time edge-aware image processing with the bilateral grid. In SIGGRAPH. ACM Press New York, NY, USA, 2007.

[22] K. Xu, Y. Li, T. Ju, S.M. Hu, and T.Q. Liu. Efficient Affinity-based Edit Propagation using KD Tree. In ACM SIGGRAPH ASIA. ACM, 2009.

[23] R. Fattal. Edge-avoiding wavelets and their applications. In ACM SIGGRAPH 2009 papers, pages 1-10. ACM, 2009.

[24] Z. Farbman, R. Fattal, D. Lischinski, and R. Szeliski. Edgepreserving decompositions for multi-scale tone and detail manipulation. In SIGGRAPH. ACM New York, NY, USA, 2008.

[25] C. Tomasi and R. Manduchi. Bilateral filtering for gray and color images. In ICCV 1998, pages 839-846, 1998.

[26] R. Fattal, M. Agrawala, and S. Rusinkiewicz. Multiscale shape and detail enhancement from multi-light image collections. ACM Transactions on Graphics, 26(3):51, 2007.

[27] W.H. Press, S.A. Teukolsky, and W.T. Vetterling. Numerical recipes in C++: the art of scientific computing. 2002.

[28] H. Samet. Applications of spatial data structures: Computer graphics, image processing, and GIS. 1990.

[29] DR Fuhrmann. Quadtree traversal algorithms for pointer-based and depth-firstrepresentations. Pattern Analysis and Machine Intelligence, IEEE Transactions on, 10(6):955-960, 1988.

[30] F. Durand and J. Dorsey. Fast bilateral filtering for the display of high-dynamic-range images. In SIGGRAPH, pages 257-266. ACM New York, NY, USA, 2002

[31] J. Wang, P. Bhat, R.A. Colburn, M. Agrawala, and M.F. Cohen. Interactive video cutout. In SIGGRAPH, pages 585-594. ACM New York, NY, USA, 2005.

[32] GM Hunter and K. Steiglitz. Operations on images using quad trees. IEEE Transactions on Pattern Analysis and Machine Intelligence, 1:145-153, 1979.

[33] D.J.R. Meagher. Octree Encoding: A New Technique for the Representation, Manipulation and Display of Arbitrary 3-D Objects by Computer. Technical Report IPL.-TR-80-111, Image Processing Labora- tory, Electrical and Systems Engineering Department, Rensseiaer Polytechnic Institute, 1980.

[34] R. Fattal, D. Lischinski, and M. Werman. Gradient domain high dynamic range compression. ACM Transactions on Graphics, 21(3):249-256, 2002.

[35] E. Reinhard, M. Stark, P. Shirley, and J. Ferwerda. Photographic tone reproduction for digital images. ACM Transactions on Graphics, 21(3):267-276, 2002.

[36] C. Xiao, Y. Nie, W. Hua, and W. Zheng. Fast multi-scale joint bilateral texture upsampling. The Visual Computer, pages 1-13, 2010.

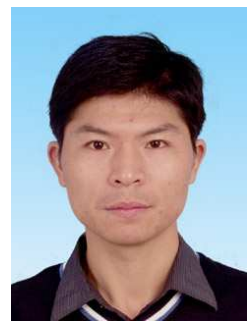

Chunxia Xiao received the BSc and MSc degrees from the Mathematics Department of Hunan Normal University in 1999 and 2002, respectively, and received the Ph.D. from the State Key Lab of CAD \& CG of Zhejiang University in 2006. He is currently an associate professor at the School of Computer, Wuhan University, China. During October 2006 to April 2007, he worked as a postdoc at the Department of Computer Science and Engineering, the Hong Kong University of Science and Technology. His research interests include image and video precessing, digital geometry processing and computational photography.

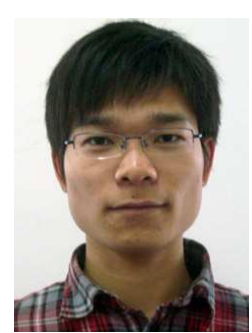

Yongwei Nie received the BS degree from the School of Computer, Wuhan University in 2009 is currently a master student at School of Computer, Wuhan University, China. His research interests include image and video editing, computational photography.

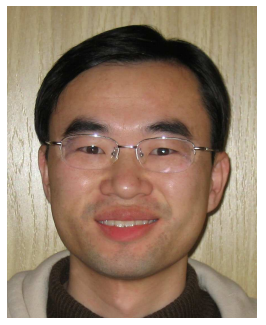

Feng Tang received the BS and MEng degrees from the Computer Science Department of Zhejiang University in 2001 and 2004, respectively. He obtained the Ph.D. degree in Computer Engineering from University of California, Santa Cruz in 2008. Since Feburary 2009, he has been with Hewlett-Packard Laboratories as a researcher in Multimedia Interaction and Understanding Lab. His research interests are in image representation, multimedia understanding, and machine learning. $\mathrm{He}$ is a member of the IEEE. 